\title{
Numerical Calculation Methods and Computer Implementation of Ordinary Differential Equation Initial Value Problem
}

\author{
ZHANG Li-juan ${ }^{1, a}$, ZHANG -Xiang ${ }^{2, b}$, GUAN Tian-ye ${ }^{3, c}$ \\ ${ }^{1}$ Mathematics and Statistical College, Baicheng Normal University, Baicheng, China, 137000 \\ ${ }^{2}$ Construction and Administration Burean of Water to Baicheng From Nen River, Baicheng, \\ China, 137000 \\ ${ }^{3}$ Jilin Province Water Conservancy and Hydropower Survey Design Institute Changchun, \\ China,130021 \\ âEmail:zhanglj4221@126.com, ${ }^{\mathrm{b} E m a i l: z h x i a n g @ 126 . c o m, ~}{ }^{\mathrm{c} E m a i l: G y e 09 @ 163 . c o m ~}$
}

Keywords: ordinary differential equation, initial value problem, numerical calculation method

\begin{abstract}
The paper solved the ordinary differential equation initial value problem on computer by MATLAB, using methods of Euler's method, improved Euler method and classical Runge-Kutta. It can draw a conclusion that simple error of Euler method principle is bigger, the calculation amount of improved Euler method is larger and the classical Runge-Kutta method has high precision stability by comparing numerical results analysis,
\end{abstract}

\section{Introduction}

We often encountered ordinary differential equation initial value problem to solve in engineering calculation and scientific research.[1] But only a small part of the initial value problems could be solved using elementary solution and the analytical solution. For the most part of them could only be solved by approximate methods.[2][3] We programed to implement initial value problems of ordinary differential equations using Euler method, improved Euler method, classical Runge-Kutta method by MATLAB.[4][5][6] We usually have to solve some initial value problems of ordinary differential equations. In fact, there are few problems which can be solved by elementary method to get analytic solution.[7] In the most case we can only use approximate method to get numerical solution. Numerical solution of differential equation is a kind of discrete method.

For the initial value problem:

$$
\left\{\begin{array}{c}
\frac{d y}{d x}=f(x, y), x \in[a, b] \\
y(a)=y_{0}
\end{array}\right.
$$

First the interval $[a, b]$ is divided into $n$ equal parts, $x_{i}=a+i h,(i=0,1,2, \cdots \cdots n)$, the step is $h=x_{i+1}-x_{i}$. Then to solve the function $y(x)$ in a series of discrete equidistant node $x_{0}<x_{1}<x_{2}<\cdots x_{n}$ to get approximate values $y_{0}<y_{1}<y_{2}<\cdots y_{n}$.

\section{Basic Theory}

Euler Method. Euler's method is the most fundamental and most simple algorithm. According to Taylor's theorem $y(x)$ in some show, $x_{n}$, we can obtain:

$$
y\left(x_{n+1}\right)=y\left(x_{n}\right)+h y^{\prime}\left(x_{n}\right)+\frac{h^{2}}{2 !} y^{\prime \prime}\left(\xi_{n}\right), \xi_{n} \in\left(x_{n}, x_{n+1}\right)
$$

When $\mathrm{h}$ is very small, the error term $R_{n}=\frac{h^{2}}{2 !} y^{\prime \prime}\left(\xi_{n}\right), \xi_{n} \in\left(x_{n}, x_{n+1}\right)$ can be omitted.

The following is approximate formula of differential equation with precise solution. 


$$
\begin{gathered}
y\left(x_{n+1}\right) \approx y\left(x_{n}\right)+h y^{\prime}\left(x_{n}\right), y^{\prime}\left(x_{n}\right)=f\left(x_{n}, y_{n}\right) \\
y\left(x_{n+1}\right) \approx y\left(x_{n}\right)+h f\left(x_{n}, y_{n}\right)
\end{gathered}
$$

Where $y_{i}$ is the approximate value of $y\left(x_{i}\right),(i=1,2, \cdots n)$, then $y_{n+1}=y_{n}+h f\left(x_{n}, y_{n}\right)$

Euler formula is:

$$
y_{n+1}=y_{n}+h f\left(x_{n}, y_{n}\right), x \in[a, b], y(a)=y_{0}
$$

Euler's method calculated $y_{n+1}$ by $y_{n}$. It is a single step. The local truncation error is $O\left(h^{2}\right)$ and truncation error is $O(h)$. It is an first order convergence method.

Improved Euler Method. In order to get a more accurate method, it uses trapezoidal integral formula instead of integral type $\int_{x_{n}}^{x_{n+1}} f(t, y(t)) d t$ Then

$$
\begin{aligned}
& \quad \int_{x_{n}}^{x_{n+1}} f(t, y(t)) d t \approx \frac{h}{2}\left\{f \left(x_{n}, y\left(x_{n}\right)+f\left(x_{n+1}, y\left(x_{n+1}\right)\right\}\right.\right. \\
& y\left(x_{n+1}\right)=y\left(x_{n}\right)+\int_{x_{n}}^{x_{n+1}} f(t, y(t)) d t \\
& y\left(x_{n+1}\right) \approx y\left(x_{n}\right)+\frac{h}{2}\left\{f \left(x_{n}, y\left(x_{n}\right)+f\left(x_{n+1}, y\left(x_{n+1}\right)\right\}\right.\right. \\
& \text { Let } y_{n} \text { to } y\left(x_{n}\right) \text { and } y_{n+1} \text { to } y\left(x_{n+1}\right) \\
& \text { Where } y_{n+1}=y_{n}+\frac{h}{2}\left\{f\left(x_{n}, y_{n}\right)+f\left(x_{n+1}, y_{n+1}\right)\right\}
\end{aligned}
$$

Trapezoid formula is a second order convergence method, but it is an implicit algorithm, so it is improved. It gets a predictor $y_{n+1}=y_{n}+h f\left(x_{n}, y_{n}\right)$ by Euler method, then it gets an approximate value by trapezoidal correction formula approximation.

$$
y_{n+1}=y_{n}+\frac{h}{2}\left\{f\left(x_{n}, y_{n}\right)+f\left(x_{n+1}, y_{n+1}\right)\right\}
$$

Predictor $\quad \tilde{y}_{n+1}=y_{n}+h f\left(x_{n}, y_{n}\right)$

Corrector $y_{n+1}=y_{n}+\frac{h}{2}\left\{f\left(x_{n}, y_{n}\right)+f\left(x_{n+1}, \tilde{y_{n+1}}\right)\right\}$

Improved Euler formula is:

$$
\left\{\begin{array}{c}
\tilde{y}_{n+1}=y_{n}+h f\left(x_{n}, y_{n}\right), \\
y_{n+1}=y_{n}+\frac{h}{2}\left\{f\left(x_{n}, y_{n}\right)+f\left(x_{n+1}, \tilde{y}_{n+1}\right)\right\}
\end{array}\right.
$$

Local truncation error is $O\left(h^{3}\right)$. The improved Euler formula is a second order convergence method.

Classic Runge-Kutta Method. Runge-Kutta method reduces data requirements to reach the same precision without higher derivative calculation.

$$
y\left(x_{n+1}\right)=y\left(x_{n}\right)+\int_{x_{n}}^{x_{n+1}} f(t, y(t)) d t
$$

According to the integral mean value theory:

$$
\int_{x_{n}}^{x_{n+1}} f(t, y(t)) d t=f\left(x_{n}+\theta h, y\left(x_{n}+\theta h\right)\right), 0 \leq \theta \leq 1
$$

The mean value $f\left(x_{n}+\theta h, y\left(x_{n}+\theta h\right)\right)$ can be expressed by a linear combination of function

$$
\text { values, } y_{n+1}=y_{n}+h \sum_{i=1}^{s} c_{i} f\left(x_{i}, y_{i}\right) \text {. }
$$

We get $S$ order Runge-Kutta scheme. According to the different requirements of precision, we calculate the undetermined coefficients. So we can get different Runge-Kutta formula. Classic fourth-order runge kutta formula is widely used in the engineering. It is a kind of absolute stability 
of the algorithm. [8][9]The local truncation error is $O\left(h^{5}\right)$.

Classic fourth-order Runge-Kutta formula is:

$$
\left\{\begin{array}{c}
y_{n+1}=y_{n}+\frac{h}{6}\left(k_{1}+2 k_{2}+2 k_{3}+k_{4}\right) \\
k_{1}=f\left(x_{n}, y_{n}\right) \\
k_{2}=f\left(x_{n}+\frac{h}{2}, y_{n}+\frac{h}{2} k_{1}\right) \\
k_{3}=f\left(x_{n}+\frac{h}{2}, y_{n}+\frac{h}{2} k_{2}\right) \\
k_{4}=f\left(x_{n}+h, y_{n}+h k_{3}\right)
\end{array}\right.
$$

\section{Implement by Computer}

$$
\left\{\begin{array}{c}
\frac{d y}{d x}=y-\frac{2 x}{y}, x \in[0,1.4], h=0.2 \\
y(0)=1
\end{array}\right.
$$

The exact solution of equation is $y=\sqrt{2 x+1}$ It performs in MATAB command window: dyfun=inline( ' $y-2 x / y$ ', $x$ '” $y$ ');

$[\mathrm{x}, \mathrm{y}]=$ ouler(dyfun,xspan,y0,h);

$[\mathrm{x}, \mathrm{y}]=$ gaijinouler(dyfun, $\mathrm{x} 0, \mathrm{y} 0, \mathrm{~h}, \mathrm{n})$;

$[\mathrm{x}, \mathrm{y}]=$ Rungekutta(dyfun, $\mathrm{x} 0, \mathrm{y} 0, \mathrm{~h}, \mathrm{n})$;

The comparison of the Euler's method, the improved Euler method and Runge-Kutta method is shown as Table 1, and the comparison curves is shown as Figure 1.

Table 1 Euler's method, the improved Euler method and Runge-Kutta method numerical solution

\begin{tabular}{|c|c|c|c|c|}
\hline$x_{i}$ & $y\left(x_{i}\right)$ & $y_{i}$ Euler & $y_{i}$ Im proveeuler & $y_{i}$ Rungekutta \\
\hline 0 & 1.00000 & 1.00000 & 1.000000 & 1.000000 \\
\hline 0.2 & 1.183216 & 1.200000 & 1.186667 & 1.183229 \\
\hline 0.4 & 1.341641 & 1.373333 & 1.348312 & 1.341667 \\
\hline 0.6 & 1.483240 & 1.531495 & 1.493704 & 1.483281 \\
\hline 0.8 & 1.612452 & 1.681085 & 1.627861 & 1.612514 \\
\hline 1.0 & 1.732051 & 1.826948 & 1.754205 & 1.732142 \\
\hline
\end{tabular}

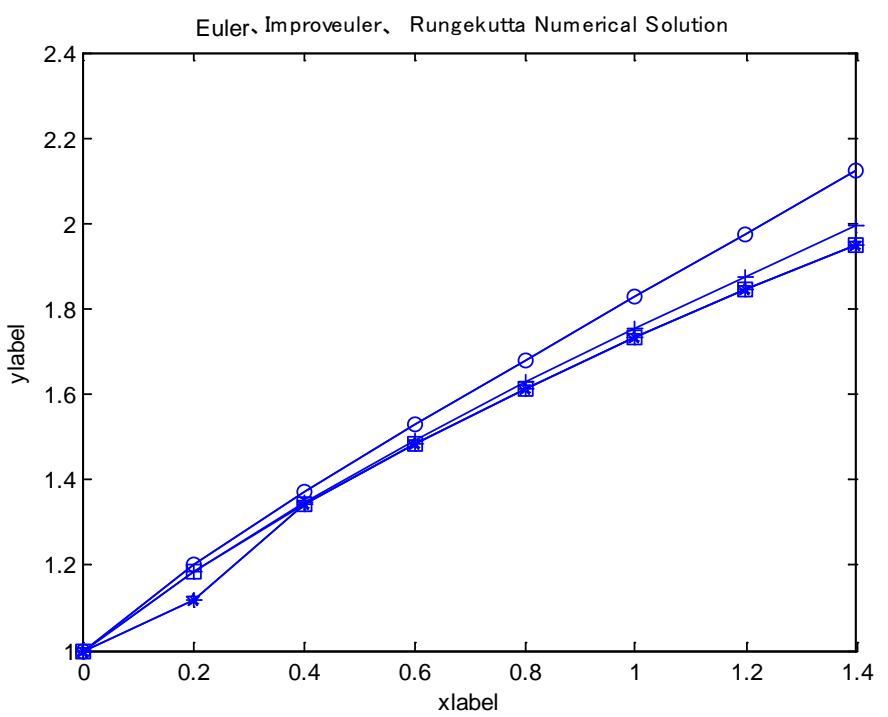

Fig. 1 The comparison curves 


\section{Conclusion}

From the comparison we can draw conclusion that improved Euler method's numerical precision is higher than Euler, and the fourth-order Runge-Kutta method is obviously better than Euler method and the improved Euler method. From the computing workload, classic fourth-order Runge-Kutta method of calculation is twice as large as improved Euler method, and four times as great as Euler method. From the standpoint error perspective, Euler method has a big error, improved Euler method's error is reduced to a certain extent. Classical fourth-order Runge-Kutta sharply reduces its error and it has high precision. So in the actual calculation, according to different engineering function, we should choose the appropriate algorithm. We not only consider method's simplicity, and but also reduce the amount of calculation. At the same time, we must insure the error in specified range. Generally in the study of ordinary differential equation initial value problem, the classical fourth-order Runge- Kutta method has high precision and is conducive to computer programming implementation. It also has good stability. It can be considered as the preferred the implementation of the algorithm.

\section{Acknowledgement}

In this paper, the research was sponsored by Fund Project of Baicheng Normal University (Project No. 2013011).

\section{References}

[1]Zhujianxin ,liyoufa .Numerical methods The third edition [M]Beijing;Higher Education Press.2012 ( 7) 67-68.

[2]Guan ye .The numerical analysis[M] Beijing;Higher Education Press. 1997.5: 122-223.

[3]Li Rong-hua ,liu bo.The numerical method for solving the differential equation The third edition[M] Beijing;Higher Education Press.2009. (3) 188-189.

[4]Zhang De-feng.Numerical analysis and application [M]Beijing:National defence industry press,2007 (1) 102-103.

[5]Practice Teaching Reform and Thinking about the "Numerical Methods for Differential Equations"Journal of Lanzhou University of Arts and Science(Natural Sciences)2014.(6)96-97.

[6]ZHANG Shu-juan The Arithmetic and Solution of Initial Value Problems of First-order Ordinary Differential Equation[J] Journal of Jingmen Technical .2007(3)52-53.

[7]WEI Ming-qiang The Comparison of Four Methods on the First Order Ordinary Differential Equation Numerical Solution .[J] Journal of Communication University of China Science and Technology 2016,23(2).154-156.

[8]ZHANG Qiu-sheng Solution of Initial Value in Ordinary Differential Equation via Single-step Method [J]Bulletin of Science and Technology2012,28(2)232-235.

[9]Huo Xiao-cheng Study on the numerical method of ordinary differential equationsournal of Juamjusi[J] Education Institute 2011(5)40-48. 